\title{
Glycosylation Regulates Prestin Cellular Activity
}

\author{
Lavanya Rajagopalan ${ }^{1,4}$ Louise E. Organ-Darling, ${ }^{6}$ Haiting Liu, ${ }^{3}$ Amy L. Davidson, ${ }^{5}$ \\ Robert M. Raphael, ${ }^{6}$ William E. Brownell, ${ }^{1,2,6}$ and Fred A. Pereira ${ }^{1,3,6}$ \\ ${ }^{1}$ Bobby R. Alford Department of Otolaryngology—Head and Neck Surgery, Baylor College of Medicine, One Baylor Plaza, \\ Houston, TX 77030, USA \\ ${ }^{2}$ Department of Neuroscience, Baylor College of Medicine, Houston, TX 77030, USA \\ ${ }^{3}$ Huffington Center on Aging and Molecular and Cellular Biology, Baylor College of Medicine, Houston, TX 77030, USA \\ ${ }^{4}$ W.M. Keck Center for Interdisciplinary Bioscience Training, Houston, TX 77005, USA \\ ${ }^{5}$ Department of Chemistry, Purdue University, West Lafayette, IN, USA \\ ${ }^{6}$ Department of Bioengineering, Rice University, Houston, TX 77005, USA
}

Received: 29 April 2009; Accepted: 12 October 2009; Online publication: 7 November 2009

\begin{abstract}
Glycosylation is a common post-translational modification of proteins and is implicated in a variety of cellular functions including protein folding, degradation, sorting and trafficking, and membrane protein recycling. The membrane protein prestin is an essential component of the membrane-based motor driving electromotility changes (electromotility) in the outer hair cell (OHC), a central process in auditory transduction. Prestin was earlier identified to possess two N-glycosylation sites (N163, N166) that, when mutated, marginally affect prestin nonlinear capacitance (NLC) function in cultured cells. Here, we show that the double mutant prestin ${ }^{\text {NN163/166AA }}$ is not glycosylated and shows the expected NLC properties in the untreated and cholesterol-depleted HEK 293 cell model. In addition, unlike WT prestin that readily forms oligomers, prestin ${ }^{\mathrm{NN} 163 / 166 \mathrm{AA}}$ is enriched as monomers and more mobile in the plasma membrane, suggesting that oligomerization of prestin is dependent on glycosylation but is not essential for the generation of NLC in HEK 293 cells. However, in the presence of increased membrane cholesterol, unlike the hyperpolarizing shift in NLC seen with WT prestin, cells expressing prestin ${ }^{\mathrm{NN} 163 / 166 \mathrm{AA}}$ exhibit a linear capacitance function. In an attempt to explain this finding, we
\end{abstract}

Electronic supplementary material The online version of this article (doi:10.1007/s10162-009-0196-5) contains supplementary material, which is available to authorized users.

Correspondence to: Fred A. Pereira - Bobby R. Alford Department of Otolaryngology-Head and Neck Surgery - Baylor College of Medicine - One Baylor Plaza, Houston, TX 77030, USA. Telephone: +1-713-7986933; fax: +1-713-7981610; email: fpereira@bcm.edu discovered that both WT prestin and prestin ${ }^{\mathrm{NN} 163 / 166 \mathrm{AA}}$ participate in cholesterol-dependent cellular trafficking. In contrast to WT prestin, prestin ${ }^{\mathrm{NN} 163 / 166 \mathrm{AA}}$ shows a significant cholesterol-dependent decrease in cellsurface expression, which may explain the loss of NLC function. Based on our observations, we conclude that glycosylation regulates self-association and cellular trafficking of prestin ${ }^{\mathrm{NN} 163 / 166 \mathrm{AA}}$. These observations are the first to implicate a regulatory role for cellular trafficking and sorting in prestin function. We speculate that the cholesterol regulation of prestin occurs through localization to and internalization from membrane microdomains by clathrin- and caveolin-dependent mechanisms.

Keywords: trafficking, membrane, glycosylation, nonlinear capacitance

\section{INTRODUCTION}

Prestin is an integral membrane protein found in outer hair cells (OHCs) of the cochlea (Zheng et al. 2000) and forms an essential component of a molecular motor based in the lateral wall of OHCs (Belyantseva et al. 2000; Liberman et al. 2002; Adler et al. 2003; Yu et al. 2006). In conjunction with the OHC membrane, prestin responds to changes in electrical potential across the membrane to elicit mechanical motion, a phenomenon called electromotility (Brownell et al. 1985; Zheng et al. 2000; Brownell 2006; Ashmore 2008). This is accompanied by a bell-shaped nonlinear capacitance (NLC) function, resulting from charge movement within, as opposed to through, the membrane (Ludwig et al. 2001; Oliver et al. 2001). This NLC is widely regarded as the electrical signature 
of electromotility and provides an effective readout of prestin function.

The lateral membrane of the OHC is a highly specialized structure adapted for electromechanical transduction at frequencies approaching $100 \mathrm{kHz}$ (Frank et al. 1999; Brownell et al. 2001; Brownell 2006; Ashmore 2008). The lateral wall of the adult OHC is low in cholesterol (Santi et al. 1994; Nguyen and Brownell 1998; Oghalai et al. 1999; Brownell and Oghalai 2000; Rajagopalan et al. 2007). This may have an impact on the modulation of prestin function, as recent observations indicate a direct and dynamic link between membrane cholesterol levels and prestin's functional and biochemical properties (Sturm et al. 2006; Rajagopalan et al. 2007).

Matsuda et al. (2004) showed that prestin is glycosylated in several cell lines and OHCs and identified residues N163 and N166 to be glycosylated. However, no role of glycosylation in prestin structure or function was demonstrated, the only obvious consequence of nonglycosylation being a small shift in voltage at peak capacitance ( $V_{\mathrm{pkc}}$; Matsuda et al. 2004). N-glycosylation of proteins is an important post-translational covalent modification that has been implicated in protein folding, sorting and trafficking, and importantly, membrane and raft localization of proteins (Huet et al. 2003; Fullekrug and Simons 2004). Based on the propensity of prestin to localize to cholesterol-rich membrane microdomains (Sturm et al. 2006; Rajagopalan et al. 2007), we investigated the possible role of glycosylation in membrane raft localization and trafficking of prestin. Our results indicate a functional and regulatory role for glycosylation in the cellular trafficking and/or internalization, particularly cholesterol-induced endocytosis, of prestin.

\section{METHODS}

Materials

Antiflotillin-1 antibody (1:250) was purchased from BD Biosciences (San Jose, CA, USA), anti-HA (1:1,000) was purchased from Cell Signaling Technology (Danvers, MA, USA), and AlexaFluor 594 goat antimouse antibody (1:800) and concanavalin AAlexaFluor 350 conjugate (working concentration $50-200 \mu \mathrm{g} / \mathrm{ml}$ ) were purchased from Molecular Probes (Carlsbad, CA, USA). Peroxidase-labeled horse antimouse antibody was obtained from Vector Laboratories (Burlingame, CA, USA). Methyl- $\beta$-cyclodextrin $(\mathrm{M} \beta \mathrm{CD})$, water-soluble cholesterol, and bovine serum albumin were obtained from Sigma (St. Louis, MO, USA). The ECL western blotting detection kit was obtained from Amersham (Piscataway, NJ, USA). N-glycopeptidase-F (PNGase-F) was obtained from
New England Biolabs (Ipswich, MA, USA). Primers were obtained from Sigma Genosys.

\section{Cloning and mutagenesis}

Gerbil prestin was cloned into the pIRES-GFP vector as a HA-tagged fusion protein (HA-prestin), as previously described (Rajagopalan et al. 2006; Sturm et al. 2006). Gerbil prestin was also cloned into the pEGFP-N1 vector as a fusion protein with GFP, as previously described (Sturm et al. 2006). A mutation A206K was engineered into the GFP sequence in the prestin-GFP plasmid to render GFP predominantly monomeric; this construct is referred to as prestin-

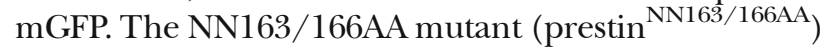
was created in both the HA-prestin and the prestinmGFP constructs using the Quikchange site-directed mutagenesis system (Stratagene, La Jolla, CA, USA) with the following primers (mutated codons are italicized): forward primer 5'GAGGAGTGGCCGCAACCGCCGG CACGGAG3'; reverse primer 5'CCTCCGTGCC GGCGGTTGCGGCCACTCCTC3'. The sequence of prestin $^{\mathrm{NN} 163 / 166 \mathrm{AA}}$ was verified using five overlapping sequencing primers. Cavl-mRFP was obtained from Dr. Ari Helenius, ETH Zurich through Addgene. The mRFP-clathrin construct was obtained from Dr. Pietro DeCamilli, HHMI, Yale University.

\section{Cell culture and transfection}

HEK 293 cell lines were maintained with Dulbecco's modified Eagle's medium with $10 \%$ FBS and $1 \%$ streptomycin and penicillin. Twenty-four hours after passage or at $\sim 50 \%$ confluence, cells were transfected with WT HA-prestin or prestin-mGFP or with HAprestin $^{\mathrm{NN163} / 166 \mathrm{AA}}$ or prestin ${ }^{\mathrm{NN163} / 166 \mathrm{AA}}$-mGFP at a 3:1 ratio with FuGene 6 (Roche, Indianapolis, IN, USA) or cotransfected (1:1 ratio) with either Cav1-mRFP or mRFP-clathrin and either WT prestin-mGFP or pres$\operatorname{tin}^{\mathrm{NN} 163 / 166 \mathrm{AA}}-\mathrm{mGFP}$ at a $3: 1$ ratio of total DNA to FuGene 6.

\section{Glycosidase treatment}

HEK 293 cells transfected with either WT HA-prestin or HA-prestin ${ }^{\mathrm{NN} 163 / 166 \mathrm{AA}}$ were harvested $48 \mathrm{~h}$ posttransfection and lysed in buffer (200 mM NaCl, $10 \mathrm{mM}$ ethylenediaminetetraacetic acid [EDTA], and $50 \mathrm{mM}$ 4-(2-hydroxyethyl)-1-piperazineethanesulfonic acid [HEPES], pH 7.4 and protease inhibitor cocktail), homogenized, and clarified by centrifugation at $1,000 \times g$ for $10 \mathrm{~min}$. Supernatants were pelleted at $110,000 \times \mathrm{g}$ for $40 \mathrm{~min}$. Pellets were solubilized $(50 \mathrm{mM}$ Tris- $\mathrm{HCl}, \mathrm{pH}$ 8.0, $1 \%$ sodium dodecyl sulfate) and denatured in glycoprotein-denaturing buffer (New England Biolabs, Ipswich, MA, USA) at $100^{\circ} \mathrm{C}$ for 
10 min before incubating with $\mathrm{N}$-glycopeptidase-F (PNGase-F) for $1 \mathrm{~h}$ at $37^{\circ} \mathrm{C}$. Proteins were separated on $10 \%$ sodium dodecyl sulfate polyacrylamide gel electrophoresis (SDS-PAGE) and transferred to a nitrocellulose membrane. The membranes were blocked with 5\% dry milk in phosphate-buffered saline with $1 \%$ Triton-X100 (PBST). The membrane was probed with anti-HA primary antibody, followed by antimouse secondary antibody at dilutions indicated above. Bands were visualized with the ECL Western blotting detection kit.

\section{Lipid raft fractionation}

Cell membranes were fractionated as previously described (Sturm et al. 2006) using the method of Vetrivel et al. (2004). Briefly, HEK 293 cells expressing HA-prestin ${ }^{\mathrm{NN} 163 / 166 \mathrm{AA}}$, either untreated, treated with $10 \mathrm{mM} \mathrm{M} \beta \mathrm{CD}$, or treated with water-soluble cholesterol for $30 \mathrm{~min}$ at $37^{\circ} \mathrm{C}$, were lysed in buffer $(0.5 \%$ Lubrol WX/17A17, Serva, 25 mM Tris-HCl, pH 7.4, $150 \mathrm{mM} \mathrm{NaCl}, 5 \mathrm{mM}$ EDTA, and $1 \mathrm{mM}$ phenylmethanesulfonyl fluoride). Membranes were separated on a $5 \%, 35 \%$, and $45 \%$ sucrose gradient. Twelve 1-mL fractions were collected and excess lipids removed by methanol/chloroform precipitation before the proteins were separated by $7.5 \%$ SDSPAGE and analyzed by Western blotting as described above.

\section{Cross-linking experiments}

Forty-eight hours after transfection with WT HAprestin or HA-prestin ${ }^{\mathrm{NN} 163 / 166 \mathrm{AA}}$, HEK 293 cells grown in 10-cm plates were either treated with $\mathrm{M} \beta \mathrm{CD}$ or water-soluble cholesterol for $30 \mathrm{~min}$ or left untreated before gentle harvesting into $1 \mathrm{~mL}$ of PBS $\mathrm{pH}$ 8.0. The cells from each plate were harvested, split into eight aliquots, pelleted $(2,000 \times \mathrm{g}$ for $5 \mathrm{~min})$, and each aliquot was incubated with various concentrations of cross-linker (bis(sulphosuccinimidyl)suberate $\left[\mathrm{BS}^{3}\right]$; 0 to $5 \mathrm{mM}$ ) for $30 \mathrm{~min}$ at room temperature. Reactions were quenched with $50 \mathrm{mM}$ Tris $\mathrm{pH} 7.5$. The samples were mixed with $8 \%$ 2X PFO sample buffer and incubated for $30 \mathrm{~min}$ at room temperature before separation on a 4-8\% Tris-glycine gel for analysis by Western blotting.

\section{Colocalization studies}

(a) Prestin cell-surface expression: HEK 293 cells grown on coverslips were transfected with HA-prestin-IRES-

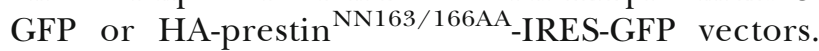
Thirty-two to $48 \mathrm{~h}$ post-transfection, cells were either left untreated or treated with $10 \mathrm{mM}$ water-soluble cholesterol or $\mathrm{M} \beta \mathrm{CD}$ for $30 \mathrm{~min}$ at $37^{\circ} \mathrm{C}$. Cells were then washed with PBS, stained with concanavalin AAlexaFluor 350 conjugate (Molecular Probes, Carlsbad, CA, USA) in PBS for $1 \mathrm{~h}$ on ice, and washed with PBS again before fixing with $4 \%$ paraformaldehyde in PBS. The cells were then stained with anti-HA antibody (1:1,200; Cell Signaling Technology, Denvers, MA, USA) in PBST for $1 \mathrm{~h}$, followed by three PBS washes and then AlexaFluor 594 goat antimouse secondary antibody (1:800; Molecular Probes, Carlsbad, CA, USA) in PBST for $1 \mathrm{~h}$ prior to PBS washes. (b) Prestin in endocytic vesicles: HEK 293 cells grown on coverslips were cotransfected with either Cav1-mRFP or mRFP-clathrin and either WT or prestin NN163/166AA mGFP. Thirty-two to $48 \mathrm{~h}$ after transfection, cells were either left untreated or treated with $10 \mathrm{mM}$ watersoluble cholesterol for $30 \mathrm{~min}$ at $37^{\circ} \mathrm{C}$. Cells were then washed with PBS before fixing with $4 \%$ paraformaldehyde for $10 \mathrm{~min}$. GFP and RFP epifluorescence were used to visualize prestin and caveolin/clathrin, respectively. For both sets of colocalization studies: Coverslips were mounted on slides with Fluoromount G antifade reagent and fluorescence captured on a Zeiss Axioplan 2 deconvolution microscope with $\times 63$ or $\times 100$ objective and analyzed with the Delta Vision Applied Precision SoftWoRx software. Quantification of prestin endocytic colocalization: The colocalization of prestin with caveolin was measured using the colocalization module included in the software package, which generates a pixel-by-pixel scatter plot of the intensities of two channels and provides the Pearson's coefficient of correlation. The Pearson's coefficient is obtained by dividing the covariance of two variables by the product of their standard deviation (SD) and reflects the strength of the linear relationship between the two variables; in this case, the intensities of GFP and RFP fluorescence.

\section{Electrophysiological measurements}

Electrophysiological data were obtained from HEK 293 cells using the whole-cell voltage-clamp technique. Cells were either voltage-clamped without treatment or treated with $10 \mathrm{mM} \mathrm{M \beta CD}$ or watersoluble cholesterol for $30 \mathrm{~min}$ at $37^{\circ} \mathrm{C}$ prior to voltageclamping. For the kinetic studies, cells were first patched, then $\mathrm{M} \beta \mathrm{CD}(100 \mu \mathrm{M}$ final concentration) or water-soluble cholesterol (1 $\mathrm{mM}$ final concentration) was added into the external solution, and NLC was recorded at time intervals of 1 to $5 \mathrm{~min}$. Our recording techniques are fully described earlier (Rajagopalan et al. 2006), but a brief description follows. Culture dishes containing transfected cells were placed on the stage of an inverted microscope (Carl Zeiss, Gottingen, Germany) under $\times 100$ magnification and extensively perfused with the extracellular solution containing $\mathrm{Ca}^{2+}$ and $\mathrm{K}^{+}$channel blockers 
(in millimolars: $100 \mathrm{NaCl}, 20 \mathrm{CsCl}, 20$ tetraethylammonium-Cl, 10 HEPES, $2 \mathrm{CoCl}_{2}, 1.47 \mathrm{MgCl}_{2}$, and 2 $\mathrm{CaCl}_{2}$ ) prior to recording. All recordings were conducted at room temperature $\left(23 \pm 1^{\circ} \mathrm{C}\right)$. Patch pipettes (quartz glass) with resistances ranging from 2 to $4 \mathrm{M} \Omega$ were fabricated using a laser-based micropipette puller (P-2000, Sutter Instrument Company, Novato, CA, USA) and filled with an intracellular solution, also containing channel blockers (in millimolars: $140 \mathrm{CsCl}$, $2 \mathrm{MgCl}, 10$ ethylene glycol tetraacetic acid, and 10 HEPES). Quartz pipettes were used as they result in lower noise levels due to the low dielectric constant, low loss factor, and extremely high volume resistivity of quartz. Cell membrane admittance was measured with the patch-clamp technique in the whole-cell mode using a DC voltage ramp with dual-frequency stimulus (Santos-Sacchi et al. 1998) from -0.14 to $0.14 \mathrm{~V}$ with a holding potential of $0 \mathrm{~V}$, and the cell parameters were calculated from the admittance as described earlier (Farrell et al. 2006). The conductance was also determined experimentally with a DC protocol, as described earlier (Rajagopalan et al. 2006).

In all representations, capacitances were normalized with respect to baseline capacitance (taken as the capacitance at $+100 \mathrm{mV}$ ) and peak capacitance (differs according to treatment) as follows:

$C_{\text {norm }}=\left[C(V)-C_{\text {baseline }}\right] / C_{\text {baseline }}$;

$C_{\text {final }}=C_{\text {norm }} / C_{\text {normpkc }}$

where $C(V)$ is the calculated capacitance at voltage $V$, $C_{\text {baseline is capacitance at baseline voltage (defined }}$ above), and $C_{\text {normpkc }}$ is equal to $C_{\text {norm }}$ at $V_{\text {pkc }}$. Charge density $\left(Q_{\max } / C_{\text {lin }}\right)$ was determined from Boltzmann fits to the data, as previously described (Rajagopalan et al. 2006).

\section{Fluorescence recovery after photobleaching}

Fluorescence recovery after photobleaching (FRAP) experiments were performed confocally as described previously (Organ and Raphael 2007) to calculate the lateral diffusion coefficient, $D$, and the immobile fraction, IF, of the molecules of interest. In brief, we monitored a segment of membrane using a circular region of interest with a diameter of $2.9 \mu \mathrm{m}$ centered along the plasma membrane. Data was collected for 410 scans with a delay of $1.5 \mathrm{~s}$ between scans, $\sim 12$ min. Bleaching was performed using an argon laser at $488 \mathrm{~nm}$ and $100 \%$ transmission. A doublebleaching protocol was used consisting of two successive bleaches, one after scan 10 and one after scan 210, to provide additional insight into the IF. A population of truly immobile molecules will be permanently photobleached during the first bleach and will not contribute to the fluorescent signal monitored during the second bleach. Thus, the second bleach should approach complete or $100 \%$ recovery. For FRAP data analysis, the Zeiss AIM physiology software quantified intensities, and the raw data was background subtracted before each bleach was individually normalized to the average of the first ten scans (scans 200-210 for the second bleach). The normalized data was used to calculate the IF and effective diffusion coefficients, and recovery curves were individually fit in Matlab (The MathWorks, Natick, MA, USA) to the solution of a one-dimensional diffusion equation. Chauvenet's criterion was applied to the calculated $D$ values to identify and remove outliers. Effective diffusion coefficients were then normalized to that of WT prestin.

\section{Cell-surface biotinylation to estimate membrane prestin population}

We used cell-surface biotinylation using the EZ-Link Sulfo-NHS-SS-Biotin reagent and kit (Thermo Fisher Scientific, Rockford, IL, USA) to tag and capture proteins expressed on the surface of HEK 293 cells transfected with WT prestin or prestin ${ }^{\mathrm{NN} 163 / 166 \mathrm{AA}}$ and either left untreated or loaded with cholesterol for $30 \mathrm{~min}$. Biotinylation was carried out according to the manufacturer's instructions; briefly, cells were washed with PBS, incubated with the biotin reagent for $30 \mathrm{~min}$ at $4^{\circ} \mathrm{C}$, and quenched before being rinsed, harvested, and lysed. The total protein in the wholecell lysate was measured using a Bradford assay and equal amounts of lysate were then passed through the streptavidin affinity column and eluted using dithiothreitol. Eluted proteins were separated by SDS-PAGE and blotted onto a nitrocellulose membrane for the detection of prestin. Band intensities on Western blots were quantitated either directly on a LI-COR Odyssey infrared imaging system using secondary antibodies supplied by the manufacturer (LI-COR Biosciences, Lincoln, NE, USA), or after chemiluminescent developing of film as described earlier in the "Methods" section using the Personal Densitometer SI with ImageQuant software (GE LifeSciences, Piscataway, NJ, USA).

\section{RESULTS}

Prestin is a glycoprotein; prestin NN163/166AA is nonglycosylated and functional in HEK 293 cells

Prestin has been shown to be $\mathrm{N}$-glycosylated in at least two mammalian cell lines (CHO and TSA) apart from OHCs (Matsuda et al. 2004). WT prestin heterologously expressed in HEK 293 cells when fractionated 
on denaturing gels migrates as multiple bands between $\sim 80$ and $\sim 120 \mathrm{kDa}$ (Fig. 1A, lane 1). Upon treatment with $\mathrm{N}$-glycosidase-F (PNGase-F) to remove $\mathrm{N}$-glycated sugars, WT prestin migrated as a single band with an apparent deglycosylated molecular mass of $\sim 80 \mathrm{kDa}$ (Fig. 1A, lane 3), close to its predicted molecular mass. We then substituted the two known $\mathrm{N}$-glycosylated residues (Matsuda et al. 2004), asparagine $(\mathrm{N})$ at positions 163 and 166, with alanine (A). The double mutant (prestin ${ }^{\mathrm{NN} 163 / 166 \mathrm{AA}}$ ) exhibited gel mobility similar to deglycosylated WT prestin, and its mobility was unchanged upon $\mathrm{N}$-glycosidase-F treatment (Fig. 1A, lanes 2 and 4).
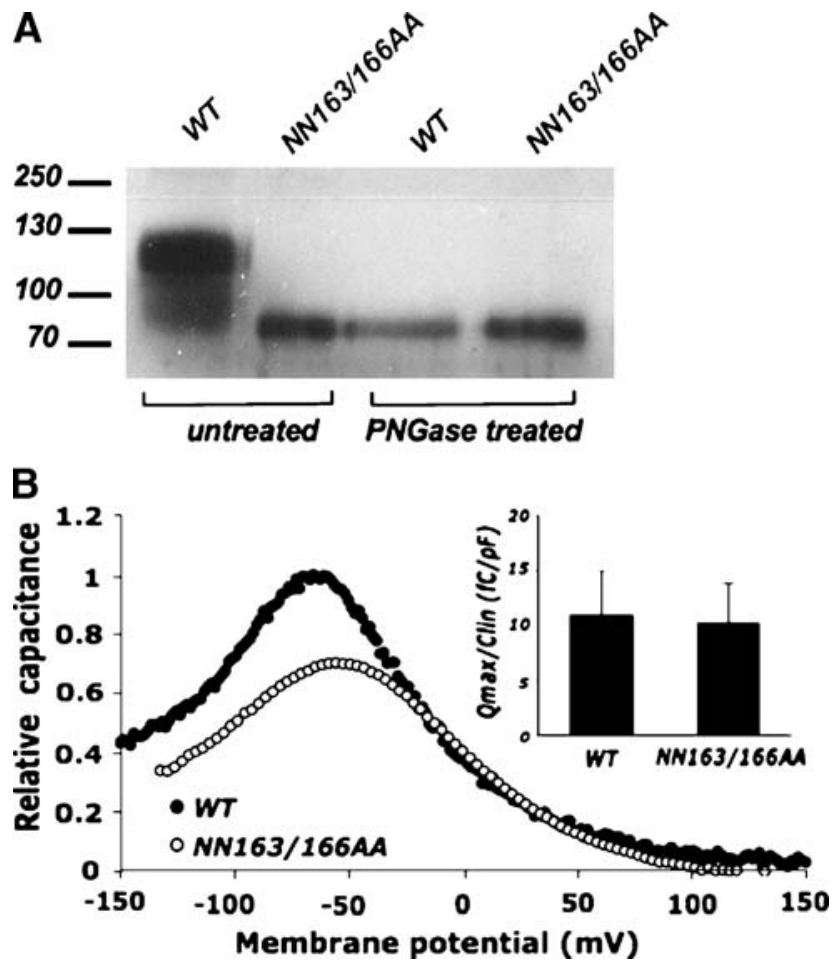

FIG. 1. Prestin is a glycoprotein and prestin ${ }^{\mathrm{NN} 163 / 166 A A}$ is functional. A Prestin is a glycosylated protein, and the prestin NN163/166AA mutant is not glycosylated in HEK 293 cells. Treatment with the glycosidase PNGase-F (to remove N-linked oligosaccharides) alters the gel migration of WT prestin. Untreated WT prestin shows a molecular weight of $\sim 120 \mathrm{kDa}$, while treatment with PNGase-F results in a band at $\sim 80 \mathrm{kDa}$, corresponding to the mass of deglycosylated prestin. Prestin NN163/166AA mutant forms only one band (at $80 \mathrm{kDa}$ ) regardless of PNGase-F treatment. B Cells transfected with NN163/166AA (white circles; $n=8$ ) exhibit a bell-shaped NLC curve with a peak slightly shifted from the WT value of $-70 \mathrm{mV}$ (black circles; $n=10$ ). Fit parameters are: for WT, $V_{\text {pkc }}=-70 \pm 18 \mathrm{mV}$; valence $(z)=0.82$; charge density $\left(Q_{\max } / C_{\text {lin }}\right)=$ $11 \pm 4 \mathrm{fC} / \mathrm{pF}$; for mutant, $V_{\mathrm{pkc}}=-55 \pm 7.5 \mathrm{mV}$; valence $(z)=0.84$; charge density $\left(Q_{\max } / C_{\text {lin }}\right)=10.3 \pm 3.5 \mathrm{fC} / \mathrm{pF}$. Representative average traces from single cells are shown. Traces have been normalized relative to maximal average NLC of WT. Inset average charge density, calculated from Boltzmann fits to NLC traces, shows no significant differences between WT prestin and prestin NN163/166AA. Error bars represent the SD.
We next measured the ability of prestin ${ }^{\mathrm{NN} 163 / 166 \mathrm{AA}}$ to confer NLC, the electrical signature of electromotility, on HEK 293 cells. The NLC properties of this double mutation were earlier demonstrated to have an insignificant depolarizing shift in $V_{\mathrm{pkc}}$, the voltage at peak capacitance, from the WT prestin value in TSA cells (Matsuda et al. 2004). We found this to also be true in HEK 293 cells where prestin ${ }^{\mathrm{NN} 163 / 166 \mathrm{AA}}$ is fully functional and exhibited an NLC peak at approximately $-55 \mathrm{mV}$, slightly depolarized from the WT value of about $-70 \mathrm{mV}$ (Fig. 1B). Charge density values remained unchanged between WT prestin and prestin $^{\mathrm{NN} 163 / 166 \mathrm{AA}}$ (Fig. 1B, inset).

\section{Prestin $^{\text {NN163/166AA }}$ expression in the plasma membrane}

As expected from the NLC activity, immunofluorescence detection of prestin ${ }^{\mathrm{NN} 163 / 166 \mathrm{AA}}$ revealed a robust expression in the plasma membrane in HEK 293 cells (Fig. 2A). Since prestin is present in membrane microdomains and several biochemical and functional properties of prestin are modulated by membrane cholesterol (Sturm et al. 2006; Rajagopalan et al. 2007), we determined if glycosylation plays a role in membrane distribution in altered cholesterol environments. Prestin ${ }^{\mathrm{NN163/166AA}}$ has a punctate distribution in the membrane, similar to WT prestin, in untreated cells (Fig. 2A, a). Qualitatively fewer and less prominent puncta were seen upon depletion of cholesterol (Fig. 2A, b) and, upon cholesterol loading, the membrane and juxtamembrane region appears to have a homogenous rather than punctate distribution of prestin ${ }^{\text {NN163/166AA }}$ (Fig. 2A, c). We next biochemically characterized prestin $^{\text {NN163/166AA }}$ expression in purified cell mem-

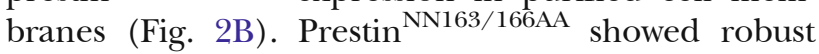
expression in membrane fractions and colocalized quite well with the membrane raft protein flotillin-1 (Fig. 2B, a, lanes 4 and 5) in a similar manner as was found for WT prestin (Sturm et al. 2006; Rajagopalan et al. 2007). Upon depletion of cholesterol, the distribution of prestin ${ }^{\mathrm{NN} 163 / 166 \mathrm{AA}}$ in the membrane fractions was not visibly altered (Fig. 2B, b), but loading cholesterol caused a decrease of prestin ${ }^{\mathrm{NN} 163 / 166 \mathrm{AA}}$ from all membrane fractions, most significantly from the membrane microdomain fractions indicated by the flotillin-1 marker (Fig. 2B, c, lanes 4 and 5).

\section{Prestin $^{\text {NN163/166AA }}$ shows lowered self-association}

We and other authors have previously demonstrated that WT prestin associates to form homodimers and is cross-linked to dimers and oligomers (Zheng et al. 


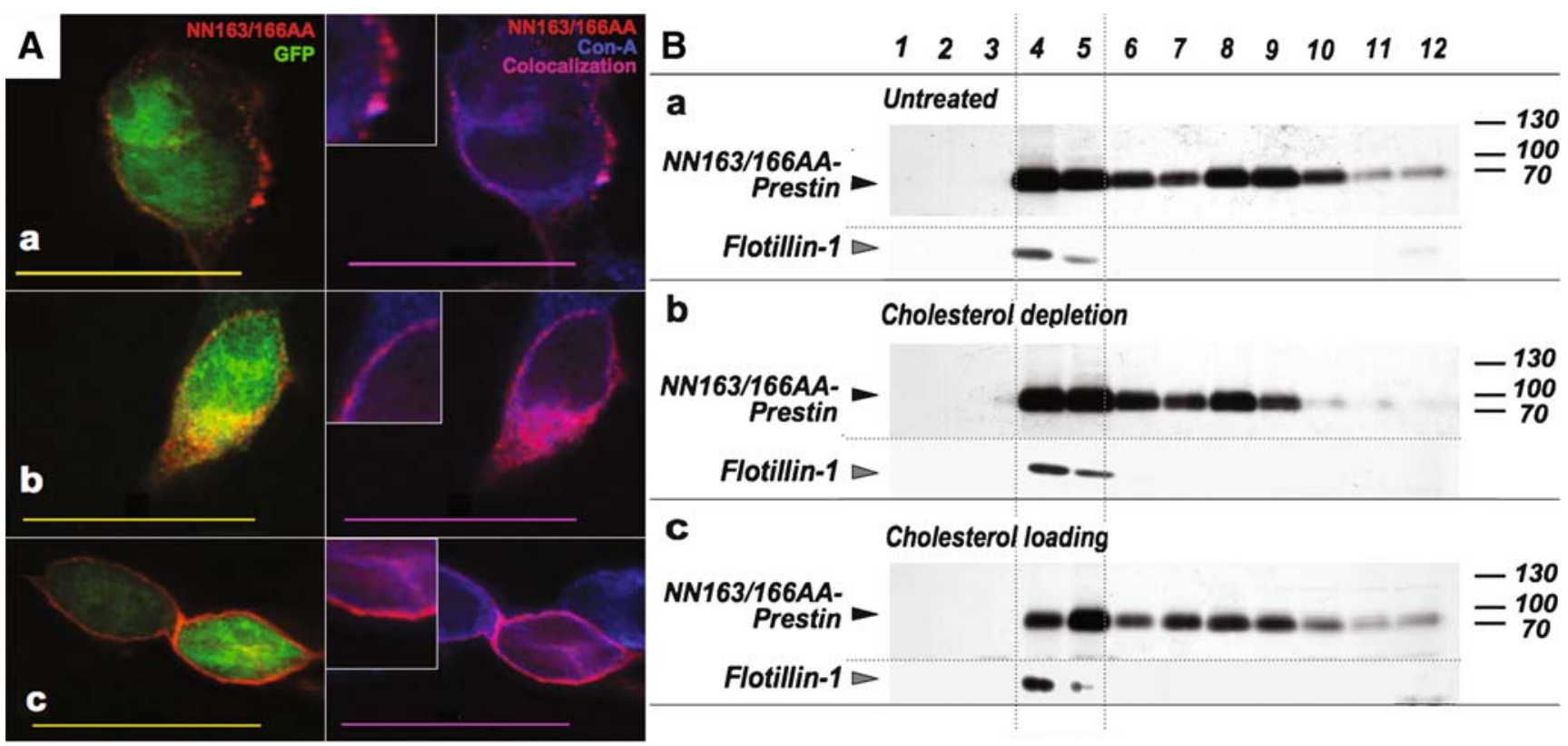

FIG. 2. Membrane distribution and localization of prestin ${ }^{\text {NN163/166AA }}$ A Immunofluorescence shows prestin fluorescence (red) colocalizes with that of concanavalin A (b/ue), a membrane marker in all cases. Epifluorescence of GFP (green), which is independently produced from the prestin plasmid as a cytoplasmic protein, has been used to identify transfected cells. a Prestin NN163/166AA exhibits punctate foci of fluorescence in the plasma membrane of HEK 293 cells. $b$ Depletion of cholesterol by $M \beta C D$ causes a decrease in the size and number of foci. $c$ Loading excess cholesterol causes an apparently homogenous distribution rather than punctate foci of prestin ${ }^{\text {NN163/166AA }}$, consistent

2006; Rajagopalan et al. 2007). We have also shown that alterations of membrane cholesterol affect the self-association properties of WT prestin (Rajagopalan et al. 2007). We thus determined if glycosylation affects prestin self-association. We used increasing concentrations of cross-linker and observed that prestin $^{\mathrm{NN} 163 / 166 \mathrm{AA}}$ does self-associate but required more cross-linker to achieve a similar level of dimer formation as WT prestin (compare lanes 2-4 in Fig. 3A-D). Cholesterol depletion did not significantly affect prestin ${ }^{\mathrm{NN} 163 / 166 \mathrm{AA}}$ self-association, while cholesterol loading enhanced self-association (compare lanes 2-4, Fig. 3A-C); an expected result because cholesterol promotes formation of microdomains and associated protein interactions, including prestin (Fullekrug and Simons 2004; Rajagopalan et al. 2007).

\section{Cholesterol loading ablates prestin NN163/166AA function}

Because cholesterol modulates various biochemical and cell biological properties of prestin ${ }^{\mathrm{NN} 163 / 166 \mathrm{AA}}$, we measured the effects of cholesterol alterations on its NLC profile. Prestin ${ }^{\text {NN163/166AA }}$ behaved similar to WT upon cholesterol depletion; $V_{\text {pkc }}$ shifted in the depolarizing direction (Fig. 4A). However, upon with the decreased raft localization see in $\mathbf{B}, \boldsymbol{c}$. Representative images from several (six to eight) cells imaged are shown. B Membrane proteins were fractionated using a sucrose density gradient. a Prestin NN163/166AA is expressed in all membrane fractions, including those containing the raft protein flotillin-1 (lanes 4 and 5). $b$ Depletion of cholesterol does not cause significant redistribution of prestinNN163/166AA in the raft fractions but $c$ loading results in a decrease of prestin NN163/166AA in all fractions, especially in the raft membrane fractions. Shown are representative blots; the experiment has been replicated $(n=3)$.

cholesterol loading, rather than exhibiting the expected hyperpolarizing shift seen for WT prestin (Rajagopalan et al. 2007), prestin ${ }^{\mathrm{NN} 163 / 166 \mathrm{AA}}$ lost its bell-shaped capacitance profile completely and demonstrated a linear capacitance function (Fig. 4A); a result expected from untransfected or mock-transfected HEK 293 cells (not shown). A comparison of average $V_{\mathrm{pkc}}$ of WT and prestin ${ }^{\mathrm{NN} 163 / 166 \mathrm{AA}}$ in different cholesterol environments is shown in Fig. 4B. To better understand the disappearance of prestin ${ }^{\mathrm{NN} 163 / 166 \mathrm{AA}}$ NLC, we carried out cholesterol manipulations at lower concentrations and at room temperature $\left(\sim 23^{\circ} \mathrm{C}\right)$ and monitored NLC as a function of time after loading. At this lower temperature and concentration, the direction of NLC changes are more gradual and can be better monitored. These kinetic studies indicate that prestin $^{\mathrm{NN} 163 / 166 \mathrm{AA}}$ does exhibit a slight hyperpolarizing shift upon cholesterol loading, albeit not to the extent seen in WT under the same conditions (Fig. 4C). However, when cholesterol was depleted from these cells, a significant depolarizing shift was observed, with the magnitude of this shift being similar in both WT and prestin ${ }^{\mathrm{NN} 163 / 166 \mathrm{AA}}$ (Fig. 4D). Furthermore, prestin ${ }^{\mathrm{NN} 163 / 166 \mathrm{AA}}$ displayed a significant $(\sim 30 \%)$ drop in charge density in the first 30 min after cholesterol loading, while the WT showed a small $(\sim 7 \%)$ decrease under the same conditions. On 


\section{Untreated}

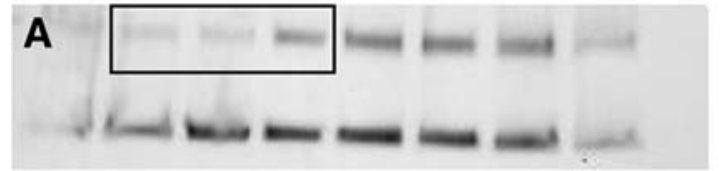

Cholesterol depletion

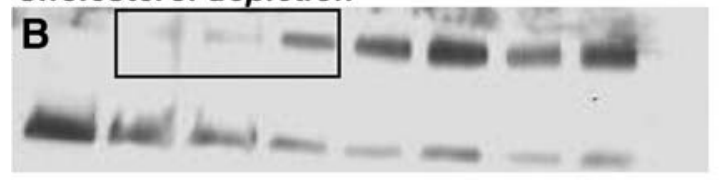

4170

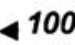

70

Cholesterol loading

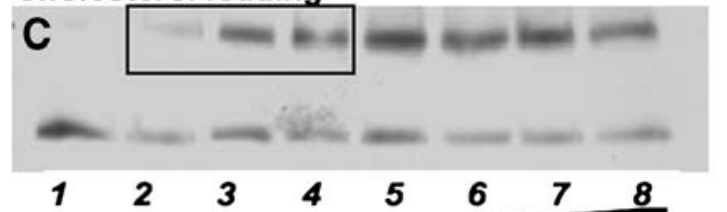

4170

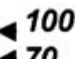

470

\section{WT untreated}

$B S^{3}$ concentration

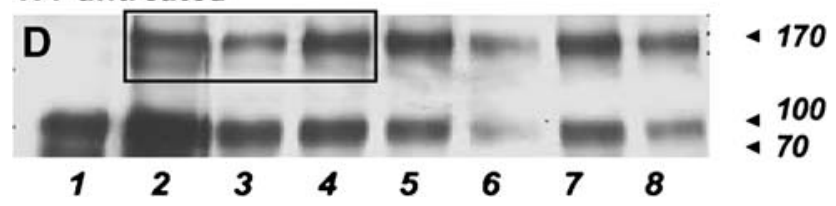

FIG. 3. Cellular cholesterol affects prestin ${ }^{\mathrm{NN} 163 / 166 \mathrm{AA}}$ self-association. A Cross-linking of prestin ${ }^{\mathrm{NN} 163 / 166 \mathrm{AA}}$ in untreated HEK 293 cells with increasing concentrations of the membrane-impermeable agent $\mathrm{BS}^{3}$ : higher concentrations of $\mathrm{BS}^{3}$ are required to cross-link the prestin $^{\mathrm{NN} 163 / 166 \mathrm{AA}}$ mutant as oligomers, compared to WT prestin (see boxed lanes, A and D). B Cross-linking of prestin NN163/166AA in cholesterol-depleted HEK 293 cells: similar concentrations of $\mathrm{BS}^{3}$ are required to cross-link prestin ${ }^{\mathrm{NN163/166AA}}$ upon cholesterol depletion as in untreated cells (boxed lanes, A and B). C Cross-linking of prestin $^{\mathrm{NN} 163 / 166 \mathrm{AA}}$ in cholesterol-loaded HEK 293 cells: oligomer bands appear at slightly lower levels of cross-linker compared to untreated cells (boxed lanes, A and C). D Cross-linking of WT prestin. In all cases, lanes 1 through 8 represent cross-linking by 0 , $0.078,0.156,0.312,0.625,1.25,2.5$, and $5 \mathrm{mM} \mathrm{BS}^{3}$, respectively. These results are obtained in cells expressing similar levels of WT prestin and prestin ${ }^{\mathrm{NN} 163 / 166 \mathrm{AA}}$ as determined by Western blotting of total prestin (data not shown). Equal loading between lanes was ensured by treating and loading equal aliquots of cells from the same dish. Cross-linking experiments have each been replicated $(n=4)$; representative blots are shown.

average, cells transfected with prestin ${ }^{\mathrm{NN} 163 / 166 \mathrm{AA}}$ exhibited a charge density reduction of $33.8 \pm 5.9 \%$ from initial values $20 \mathrm{~min}$ after the addition of cholesterol, compared with no significant change in WT. Additionally, a dramatic decrease in linear capacitance is seen with cholesterol loading in cells transfected with prestin ${ }^{\mathrm{NN} 163 / 166 \mathrm{AA}}$, while the WT showed no decrease in linear capacitance.

Prestin undergoes cholesterol-dependent changes in trafficking

Seeking an explanation for the anomalous functional response of prestin ${ }^{\mathrm{NN} 163 / 166 \mathrm{AA}}$ to cholesterol loading, we determined if the expected trafficking and endocytic recycling of prestin, a membrane protein, to the plasma membrane was dependent on and altered in the different cholesterol environments. We analyzed the degree of colocalization of caveolin-mRFP and mRFP-clathrin with WT prestin-mGFP and to prestin $^{\mathrm{NN} 163 / 166 \mathrm{AA}}$-mGFP in untreated and cholesterolloaded cells (Fig. 5). In untreated cells where both prestins are functional, each prestin had extensive colocalization with the clathrin and caveolin vesicles and in membrane localized, caveolin-rich microdomains. In addition, there was a significantly higher colocalization of both clathrin and caveolin with prestin $^{\mathrm{NN} 163 / 166 \mathrm{AA}}$ than with WT prestin. Loading cholesterol, which is known to stimulate endocytosis, caused a significant increase in WT prestin colocalization with both caveolin and clathrin, suggesting that prestin undergoes cholesterol-dependent endocytosis

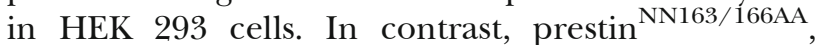
which was already highly colocalized with caveolin, did not show any further increases in colocalization upon cholesterol loading, but had a significant decrease in clathrin colocalization (Fig. 5).

Prestin NN163/166AA $^{\text {exhibits a lower lateral }}$ mobility and an enhanced internalization from the cell surface following cholesterol loading

We next determined if there were concomitant changes in prestin at the membrane and cell surface upon cholesterol manipulations. Since cholesterol loading may alter the mobility of proteins within the plasma membrane, we measured the lateral mobility of WT prestin-mGFP and prestin ${ }^{\mathrm{NN163} / 166 \mathrm{AA}}-\mathrm{mGFP}$ in the membrane using FRAP. In untreated cells, pres$\operatorname{tin}^{\mathrm{NN} 163 / 166 \mathrm{AA}}$-mGFP demonstrated a significantly higher lateral diffusion than WT prestin-mGFP in the steady state (Fig. 6A). Loading cholesterol did not alter the WT prestin-mGFP diffusion; however, cholesterol loading resulted in a significantly lower lateral diffusion of prestin ${ }^{\mathrm{NN} 163 / 166 \mathrm{AA}}$-mGFP (Fig. 6A). Analysis of the IF showed a higher second bleach IF for prestin $^{\mathrm{NN} 163 / 166 \mathrm{AA}}$-mGFP after cholesterol loading (Fig. 6B), which suggests detection of a slow-moving intracellular pool of prestin ${ }^{\mathrm{NN} 163 / 166 \mathrm{AA}}$-mGFP (Supplemental Fig. S1). There was no change in the IF for WT prestin-mGFP in cholesterol-loaded versus untreated cells (Fig. 6B). We next analyzed prestin levels at the cell surface using a biotinylation assay to quantitate WT prestin and prestin ${ }^{\mathrm{NN} 163 / 166 \mathrm{AA}}$ populations at the plasma membrane in untreated and cholesterol-loaded cells. We compared the population of WT and prestin ${ }^{\mathrm{NN} 163 / 166 \mathrm{AA}}$ in cholesterol-loaded cells relative to the corresponding untreated cells. On average, $\mathrm{WT}$ prestin showed no significant changes in 

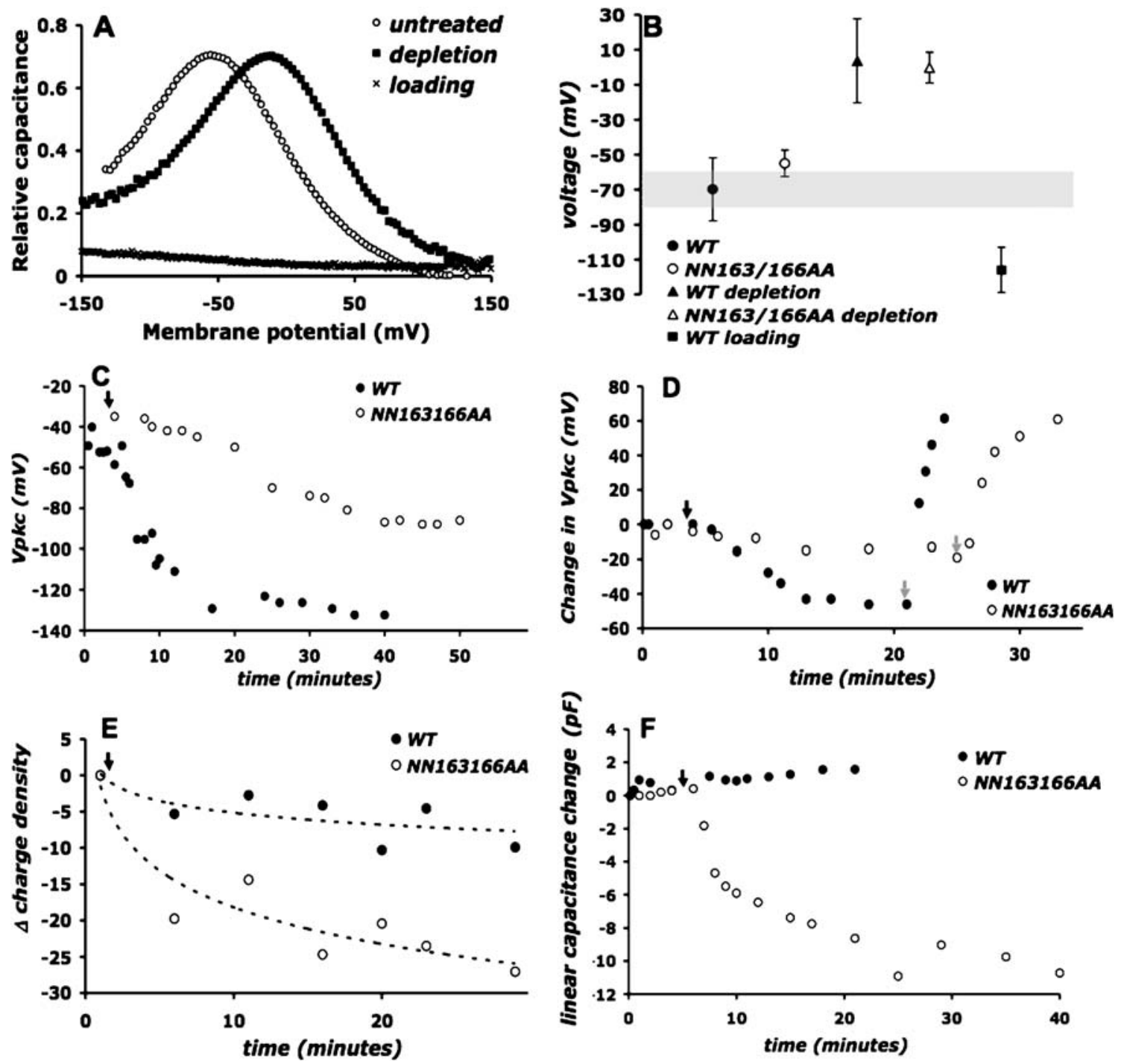

FIG. 4. Cholesterol affects membrane capacitance and peak voltage of HEK 293 cells expressing prestin ${ }^{\text {NN163/166AA }}$. A Untreated cells expressing prestin NN163/166AA exhibit a bell-shaped NLC function (black squares; $n=8$ ). Fit parameters: $V_{\mathrm{pkc}}=-55 \pm 7.5 \mathrm{mV}$; valence $(z)=0.84$; charge density $\left(Q_{\max } / C_{\text {lin }}\right)=10.3 \pm 3.5 \mathrm{fC} / \mathrm{pF}$. The NLC peak shifts towards depolarized voltages, similar to the WT trend (Sturm et al. 2006; Rajagopalan et al. 2007), in cells depleted of cholesterol (white squares; $n=6$ ) in comparison to untreated cells. Fit parameters: $V_{\mathrm{pkc}}=-0.16 \pm 8.9 \mathrm{mV}$; valence $(z)=0.81$; charge density $\left(Q_{\max } / C_{\text {lin }}\right)=7.4 \pm 9.3 \mathrm{fC} / \mathrm{pF}$. However, cells loaded with cholesterol exhibit loss of the bell-shaped capacitance curve and exhibit linear capacitance (multiplication sign; $n=6$ ) similar to untransfected and mock-transfected HEK 293 cells. Representative average traces from single cells are shown. Traces have been normalized relative to baseline and peak voltage. B Mean voltage at peak capacitance $\left(V_{\text {pkc }}\right)$ for WT prestin and prestin ${ }^{\text {NN163/166AA in }}$ HEK 293 cells. All parameters are similar between WT and prestin $^{\mathrm{NN} 163 / 166 \mathrm{AA}}$, except in the case of cholesterol loading. Error

bars represent SD. The gray box represents the normal range of $V_{\mathrm{pkc}}$ for WT prestin. C Change in $V_{\text {pkc }}$ in representative single cells transfected with WT (black circles) or prestin NN163/166AA (white circles) upon cholesterol loading. D Change in $V_{\mathrm{pkc}}$ in representative single cells transfected with WT (black circles) or prestin NN163/166AA (white circles) upon cholesterol loading (black arrow) followed by depletion (gray arrows). $\mathbf{E}$ Change in charge density in representative single cells transfected with WT (black circles) or prestin NN163/166AA (white circles) upon cholesterol loading. Cells transfected with prestin ${ }^{\mathrm{NN} 163 / 166 \mathrm{AA}}$ exhibited an average charge density reduction of $33.8 \pm 5.9 \%$ from initial values $20 \mathrm{~min}$ after the addition of cholesterol, compared with no significant change in WT. F Change in linear capacitance in representative single cells transfected with WT (black circles) or prestin NN163/166AA (white circles) upon cholesterol loading. In $\mathbf{C}-\mathbf{F}$, the black arrow indicates time of loading and the gray arrow indicates the time of depletion using $M \beta C D$. Data from representative single cells are shown; the experiments have been repeated in four cells for each treatment. 
cell-surface expression in cholesterol-loaded cells (Fig. 6C). However, prestin ${ }^{\mathrm{NN} 163 / 166 \mathrm{AA}}$ showed a significant $(p$ value $<0.05)$ decrease in cell-surface labeling upon cholesterol loading (Fig. 6C), which is consistent with enhanced internalization and loss of NLC activity. A representative blot of cell-surface WT and prestin ${ }^{\mathrm{NN} 163 / 166 \mathrm{AA}}$ protein levels in untreated and loaded cells is shown in Fig. 6D.

\section{DISCUSSION}

N-linked glycosylation of proteins in the endoplasmic reticulum and Golgi apparatus vastly increases the structural complexity of membrane proteins. Several cellular functions have been attributed to protein glycosylation: protein folding, quality control and degradation, protein sorting, trafficking and translocation, and raft or membrane microdomain localization (Helenius and Aebi 2001). We have previously shown that membrane cholesterol modulates prestin function as well as overall auditory function (Sturm et al. 2006; Rajagopalan et al. 2007), underlining the dynamic interplay between prestin and the membrane in the generation of $\mathrm{OHC}$ electromotility. The involvement of N-glycosylation in protein sorting and raft interactions of some membrane proteins prompted the present investigation of the role of glycosylation in prestin function.

We have confirmed that prestin is a glycoprotein in HEK 293 cells and the double mutation of putative $\mathrm{N}$ glycosylation sites results in prestin ${ }^{\mathrm{NN163/166AA}}$ being nonglycosylated, which is consistent with initial observations in other cell lines (Matsuda et al. 2004).

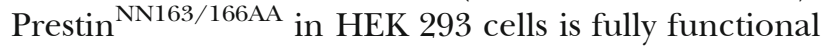
and has a bell-shaped NLC function similar to WT, but with a slightly depolarized peak consistent with the previous observation in CHO and TSA cells (Matsuda et al. 2004). Because N-glycosylation sites of transmembrane (TM) helices are generally positioned on the luminal or extracellular surface of membranes and the flipping of TM helices (along with their attached sugars) to an intracellular compartment are without precedent, these data minimize the proposed ten-pass TM topology model (Navaratnam et al. 2005) and lend further support for the modified 12-pass TM model for prestin (Zheng et al. 2001; Matsuda et al. 2004; Deak et al. 2005).

We observed that prestin ${ }^{\mathrm{NN} 163 / 166 \mathrm{AA}}$, like deglycosylated WT prestin, exists to a greater degree as monomers. Indeed, cross-linking studies indicate that higher amounts of $\mathrm{BS}^{3}$ cross-linker are required to trap prestin ${ }^{\mathrm{NN} 163 / 166 \mathrm{AA}}$ dimers when compared to WT prestin (Rajagopalan et al. 2007), suggesting that prestin $^{\mathrm{NN} 163 / 166 \mathrm{AA}}$ molecules are more distant from each other. In addition, FRAP analysis of prestin lateral mobility in HEK 293 cells indicates that prestin $^{\mathrm{NN} 163 / 166 \mathrm{AA}}$ is more mobile in the membrane with significantly higher diffusion than WT prestin. An interpretation of these data is that prestin ${ }^{\text {NN163/166AA }}$ exists predominantly in a monomeric population compared to WT prestin. This would mean that glycosylation is important for prestin self-association and oligomerization, potentially resulting from stabilizing interactions within the cell-surface glycocalyx, a known effect of glycosylation (Trombetta and Helenius 1998; Helenius and Aebi 2001; Fullekrug and Simons 2004).

Because membrane cholesterol affects prestin selfassociation and localization to rafts, as well as its membrane distribution and function (Rajagopalan et al. 2007), we assessed the cellular and functional consequence of a lack of glycosylation of prestin. Prestin $^{\text {NN163/166AA }}$ has the expected expression in HEK 293 cell membrane fractions, including membrane rafts, and cholesterol depletion did not significantly change its steady-state distribution among the different membrane fractions. These results are in contrast to cholesterol depletion of WT prestin, which redistributes out of membrane microdomains to the other membrane fractions (Sturm et al. 2006; Rajagopalan et al. 2007). Functionally, the NLC curve of prestin ${ }^{\mathrm{NN} 163 / 166 \mathrm{AA}}$ is similar to WT prestin in untreated cells and the peak of prestin ${ }^{\text {NN163/166AA }}$ NLC shifts to depolarized voltages in cells depleted of cholesterol, similar to WT behavior. These results and our findings that prestin ${ }^{\mathrm{NN} 163 / 166 \mathrm{AA}}$ is enriched as monomers and more mobile in the plasma membrane suggest that oligomerization of prestin is not essential for the generation of NLC in HEK 293 cells in normal- and low-cholesterol environments and perhaps in the OHC lateral wall, which is low in cholesterol (Rajagopalan et al. 2007).

In contrast, the NLC of prestin ${ }^{\mathrm{NN} 163 / 166 \mathrm{AA}}$ is markedly different from WT prestin in one aspect: loading excess cholesterol eliminates voltage-dependent capacitance in prestin ${ }^{\mathrm{NN} 163 / 166 \mathrm{AA}}$. Our kinetic studies indicate that this is due to a decrease in magnitude (charge density) of the NLC function and not due to an extreme shift in $V_{\mathrm{pkc}}$. The significant decrease in linear 
A
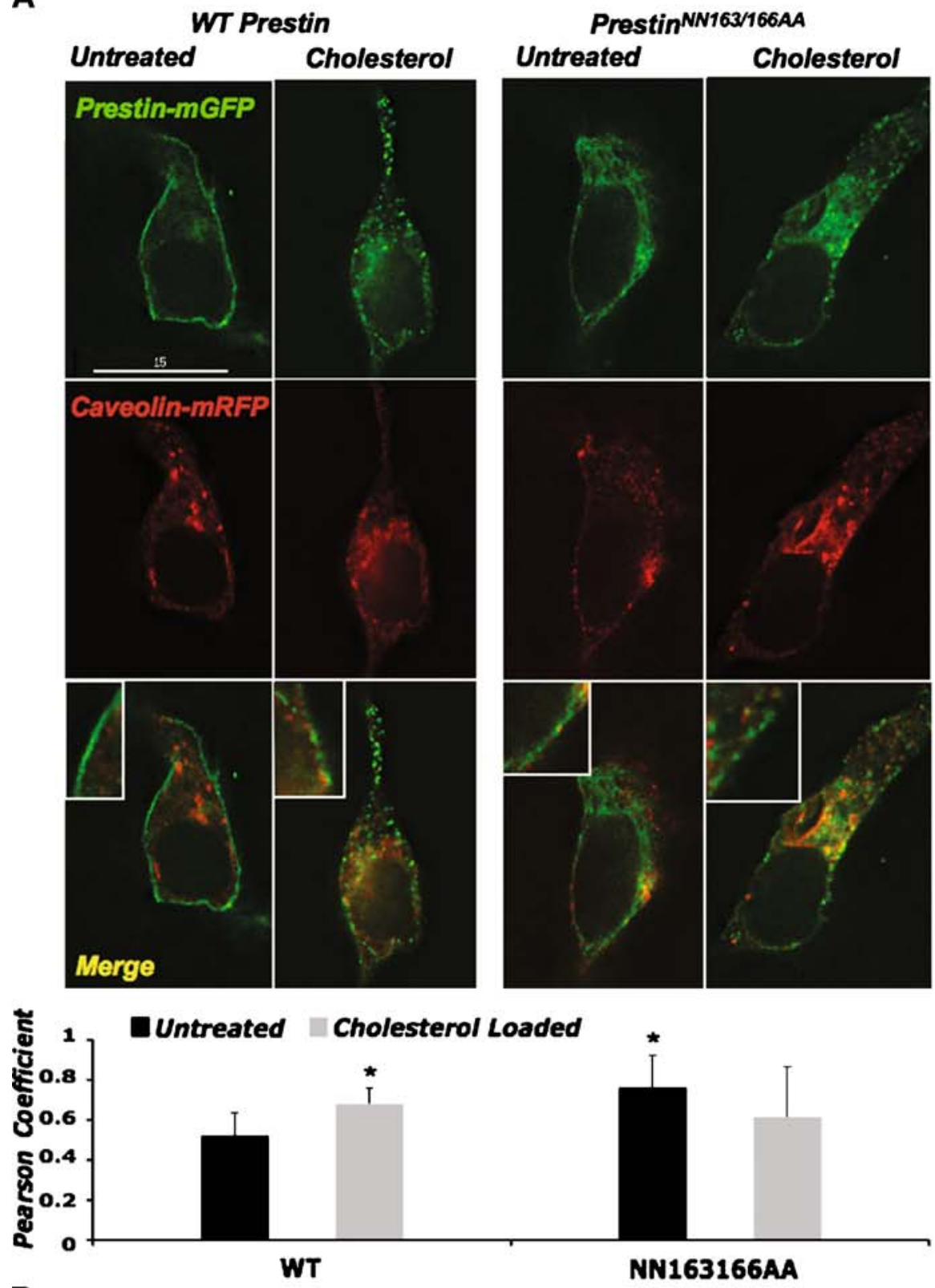

B
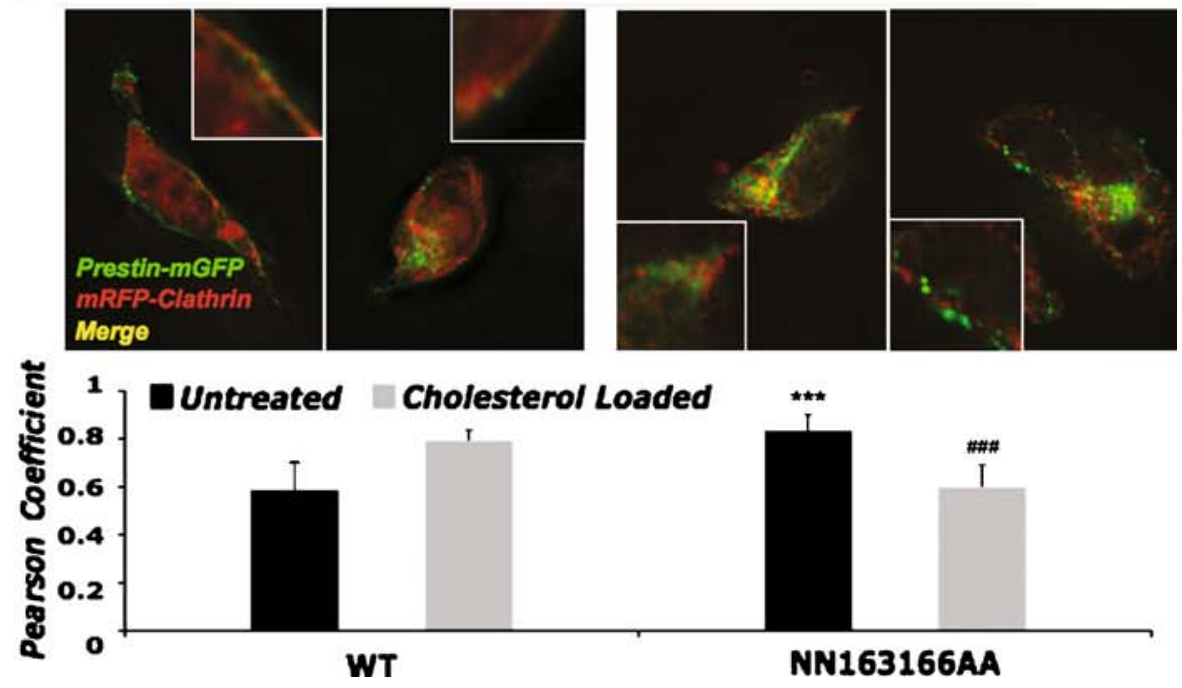
FIG. 5. WT prestin and prestin ${ }^{\mathrm{NN} 163 / 166 \mathrm{AA}}$ colocalize with caveolin and clathrin vesicles. A top deconvolution images of WT prestinmGFP and prestin ${ }^{\mathrm{NN} 163 / 166 \mathrm{AA}}$-mGFP in HEK 293 cells show colocalization of prestin (green) with Cav1-mRFP (red); bottom the Pearson's coefficient of correlation between prestin and caveolin fluorescence indicates a significant increase in caveolin colocalization with WT prestin-mGFP upon cholesterol loading. The prestin ${ }^{\mathrm{NN} 163 / 166 \mathrm{AA}}-\mathrm{mGFP}$, which colocalizes more strongly than WT in untreated cells, does not show significant differences in colocalization upon cholesterol loading. ${ }^{*} p<0.05$, statistical significance in comparison to WT untreated as determined by Student's $t$ tests. Data represent the mean $\pm \mathrm{SD}$ from five to seven cells per group. B top deconvolution images of WT prestin-mGFP and prestin ${ }^{\text {NN163/166AA }}$ mGFP in HEK cells show colocalization of prestin (green) with mRFPclathrin (red); bottom the Pearson's coefficient of correlation between prestin and clathrin fluorescence indicates a significant increase in clathrin colocalization with WT prestin-mGFP upon cholesterol loading. Prestin ${ }^{\mathrm{NN} 163 / 166 \mathrm{AA}}-\mathrm{mGFP}$ colocalizes more strongly with clathrin than WT in untreated cells, but this colocalization decreases significantly upon cholesterol loading. ${ }^{* *} p<0.01$; ${ }^{* * *} p<0.005$, statistical significance (determined by Student's $t$ tests; comparison to WT untreated) or ${ }^{\# \# \#} p<0.005$, statistical significance (determined by Student's $t$ tests; comparison to prestin NN163/166AA untreated). Data represent the mean \pm SD from five to six cells per group.

capacitance seen upon cholesterol loading in cells expressing prestin ${ }^{\mathrm{NN} 163 / 166 \mathrm{AA}}$ indicates a loss of cellsurface area. This, along with the drop in charge density and the decreased total level of prestin ${ }^{\mathrm{NN} 163 / 166 \mathrm{AA}}$ expression in the plasma membrane upon cholesterol loading, is suggestive of endocytosis triggered by cholesterol loading. The loss of linear capacitance and decrease in charge density is not seen in WT, possibly due to efficient cell-surface recycling of prestin endocytic vesicles.

The main difference between WT prestin and prestin $^{\mathrm{NN} 163 / 166 \mathrm{AA}}$ appears to be in their localization in the plasma membrane and in their responses to increased membrane cholesterol, a known effector of endocytosis. One key function of $\mathrm{N}$-glycosylation is in the regulation of protein sorting and trafficking (Helenius and Aebi 2001; Huet et al. 2003). Thus, an explanation for the ablation of prestin ${ }^{\text {NN163/166AA }}$ NLC following cholesterol loading is that cholesterol promotes prestin endocytosis; prestin $^{\mathrm{NN} 163 / 166 \mathrm{AA}}$ should then exhibit changes in trafficking and/or be internalized more readily from high-cholesterol membranes compared to WT prestin. We, therefore, investigated the colocalization of WT prestin and prestin $^{\text {NN163/166AA }}$ with clathrin and caveolin in different cholesterol environments. Prestin ${ }^{\text {NN163/166AA }}$ exhibits a much higher degree of colocalization with clathrin and caveolin vesicles than does WT prestin. This may explain the consistent presence of prestin ${ }^{\mathrm{NN} 163 / 166 \mathrm{AA}}$ in all membrane fractions and/or substantiate the possibility for its increased trafficking or sorting. The high caveolin colocalization and decreased clathrin colocalization of prestin ${ }^{\mathrm{NN} 163 / 166 \mathrm{AA}}$ in cholesterol-loading conditions indicates that it is predomi- nantly internalized by a caveolin-dependent mechanism in high-cholesterol environments. Importantly, WT prestin also has a significant increase in colocalization with clathrin and caveolin upon cholesterol loading, indicating that prestin trafficking is dynamic and occurs via both clathrin- and caveolin-dependent mechanisms in HEK 293 cells. Further studies will be necessary to determine if prestin endocytosis is restricted to a dependence on dynamin for vesicle excision or may participate in other clathrin-independent endocytic pathways (Mayor and Pagano 2007).

Additional evidence that both WT prestin and prestin $^{\mathrm{NN163/166AA}}$ participate in cholesterol-dependent endocytosis comes from measurements of lateral mobility using FRAP. There is a significantly larger IF from the first bleach for both proteins upon cholesterol loading (compared to control), suggesting increased sequestering/immobilization of prestin, as in cholesterol-induced endocytic vesicles. After the second bleach, WT prestin shows no further increase in the IF upon cholesterol loading (compared to untreated), suggesting that this endocytic pool has reached steadystate recycling. However, in the case of cholesterol loading and prestin ${ }^{\mathrm{NN} 163 / 166 \mathrm{AA}}$, there is still a significantly higher IF after the second bleach. This significant IF is paralleled by a concomitantly low lateral diffusion, possibly indicating that a significant fraction of prestin ${ }^{\mathrm{NN} 163 / 166 \mathrm{AA}}$ is still being endocytosed (Kenworthy et al. 2004). Prestin ${ }^{\text {NN163/166AA }}$ may be sequestered into a nonmobile membrane or juxtamembrane compartment, i.e., into caveolin vesicles or caveolin-rich membrane microdomains, reflected as loss of punctate distribution upon cholesterol loading. This conclusion is borne out by measurements of cell-surface populations of WT prestin and prestin ${ }^{\mathrm{NN} 163 / 166 \mathrm{AA}}$, which show a significant decrease in prestin ${ }^{\mathrm{NN} 163 / 166 \mathrm{AA}}$ at the cell surface following cholesterol loading. Such a decrease is not seen in the case of WT prestin. These findings point to the possibility that the enriched fluorescence seen at the membrane with cholesterol loading is representative of a population just below, not at, the cell surfacepossibly in endocytic vesicles triggered by cholesteroldependent mechanisms. This explanation implies that a "threshold" amount of functional prestin is required in the membrane to generate measurable NLC; the relationship between prestin levels and magnitude of NLC has not been studied in detail to date.

In conclusion, $\mathrm{N}$-glycosylation of prestin is a mechanism by which the cell regulates the level of prestin trafficking to and from the plasma membrane. Our findings correlate well with another study investigating the effect of cholesterol manipulations on the magnitude of prestin-associated charge movement (Sfondouris et al. 2008). In addition to a hyperpolarizing shift, the study demonstrates that there is an associated small decrease in charge density for WT 
A

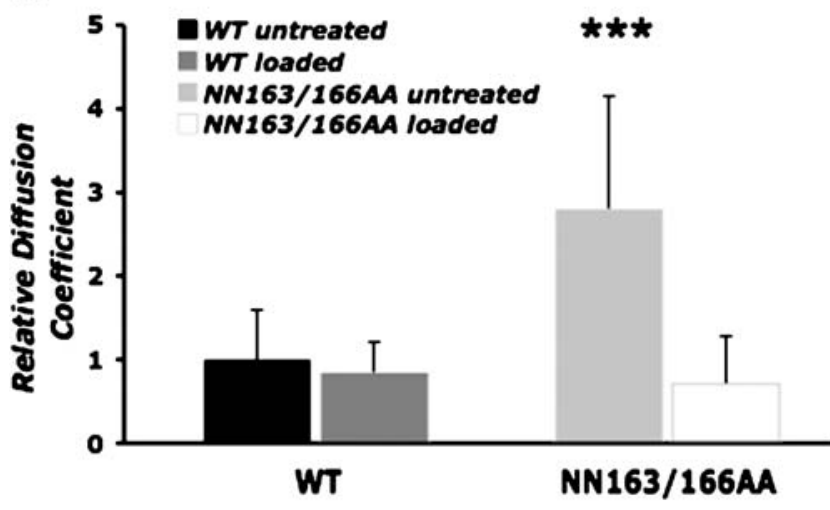

C

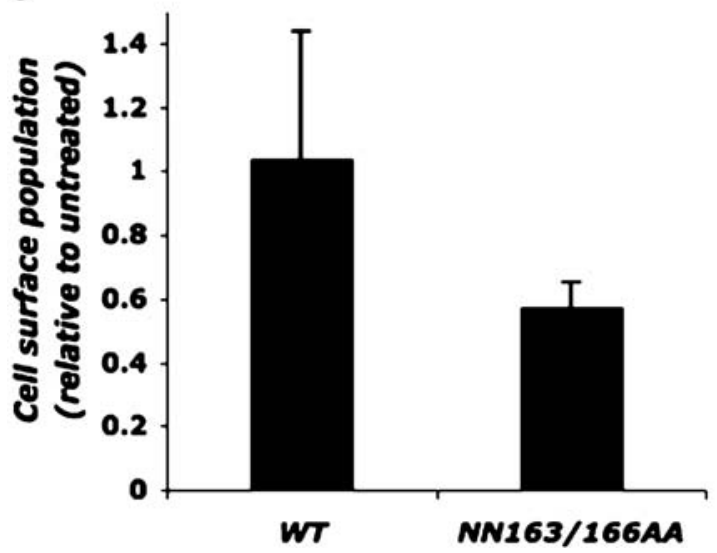

FIG. 6. Prestin ${ }^{\mathrm{NN} 163 / 166 \mathrm{AA}}$ has higher membrane mobility; cholesterol loading causes a decrease in mobility and cell-surface population. A The relative diffusion of prestin ${ }^{\mathrm{NN} 163 / 166 \mathrm{AA}}$ is significantly higher than that of WT. Cholesterol loading has a minimal effect of the lateral diffusion of WT prestin, but dramatically reduced the lateral diffusion of prestin NN163/166AA ${ }^{\text {. All diffusion measurements }}$ have been normalized to that of WT. B In the first bleach, both constructs show an increase in IF upon loading, indicative of an increase in endocytotic vesicles/caveolae. However, with this first IF effectively discounted by the first bleach, the second bleach shows no change in the IF of WT prestin upon cholesterol loading, while mutant prestin ${ }^{\mathrm{NN} 163 / 166 \mathrm{AA}}$ exhibits a large increase in IF. Values are shown as the mean $\pm \mathrm{SD} .{ }^{*} p<0.05,{ }^{* *} p<0.01,{ }^{* * *} p<0.005$; significance was determined using analysis of variance with Tukey's honestly significant difference test for diffusion measurements and Student's $t$ tests for IF data. For $\mathbf{A}$ and $\mathbf{B}$, sample sizes are as follows:

prestin upon cholesterol loading. This decrease in charge density, unlike the peak shift, is not immediately reversible, indicating that separate mechanisms are responsible for each phenomenon. Our finding that prestin $^{\mathrm{NN} 163 / 166 \mathrm{AA}}$ undergoes cholesterol-dependent endocytosis explains the associated decrease in charge density. Our findings suggest that, both during development and during conditions of cholesterolemia or lipid storage disease states (Tami et al. 1985; Saito et al. 1986; Sikora et al. 1986; Preyer et al. 2001; Evans et al. 2006; Yanjanin et al. 2009), glycosylation of prestin would play a vital role in regulating cell-surface expression of prestin to effect and maintain normal hearing. Based on our observations, we conclude that glycosylation
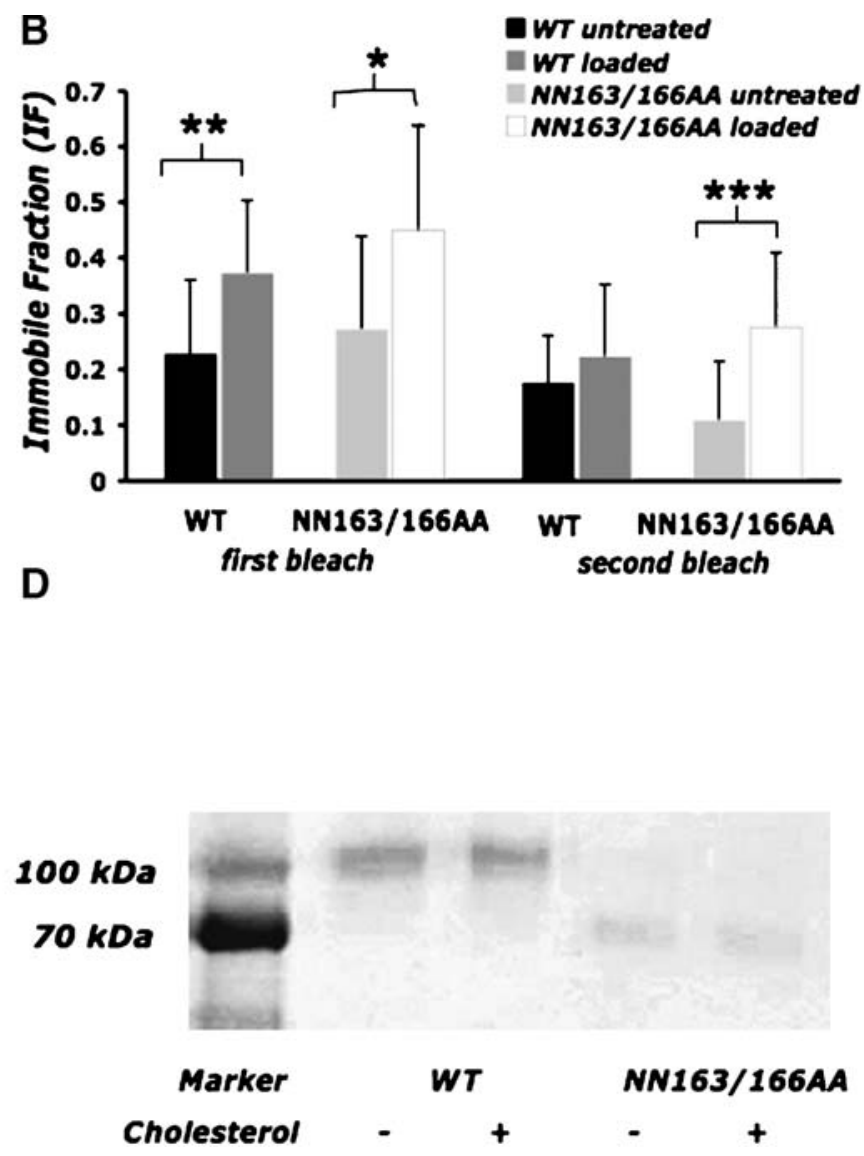

WT untreated, $n=21$; WT loaded, $n=10$; mutant untreated, $n=12$; mutant loaded, $n=9$. C The relative cell-surface population of WT prestin after cholesterol loading (compared to untreated cells) is unchanged on average, while prestin ${ }^{\mathrm{NN} 163 / 166 \mathrm{AA}}$ shows a significant $(>40 \% ; p<0.05)$ decrease in cell-surface population in cholesterolloaded cells when compared to untreated cells. Plotted are average relative cell-surface populations of cholesterol-loaded HEK 293 cells (compared to untreated cells expressing the same prestin variant), calculated from three separate experiments. Comparisons between WT prestin and prestin NN163/166AA have not been made due to intrinsic expression differences between the WT and mutant plasmids. D Representative blots of WT and prestin NN163/166AA cellsurface populations in untreated and cholesterol-loaded cells. Protein concentrations were determined for each sample in order to load equal amounts of whole-cell lysates for fractionation.

regulates self-association and cellular trafficking of prestin, most likely by regulating its localization to and/or internalization from membrane microdomains by clathrin- and caveolin-dependent mechanisms.

\section{ACKNOWLEDGEMENTS}

This work was funded by DC00354 (NIDCD; to WEB and FAP) and DC008134 (NIDCD; to FAP and RMR) and by a research grant from the Deafness Research Foundation to LR. LR was also supported by a NIH T90 postdoctoral training fellowship DK070121 through the Keck Center for Interdisciplinary Bioscience. LEO was supported by a NIDCD predoctoral training grant DC008058. 


\section{REFERENCES}

Adler HJ, Belyantseva iA, Merritt RCJ, Frolenkov GI, Dougherty GW, KACHAR B (2003) Expression of prestin, a membrane motor protein, in the mammalian auditory and vestibular periphery. Hear Res 184:27-40

Ashmore J (2008) Cochlear outer hair cell motility. Physiol Rev $88: 173-210$

Belyantseva IA, Adler HJ, Curi R, Frolenkov GI, Kachar B (2000) Expression and localization of prestin and the sugar transporter GLUT-5 during development of electromotility in cochlear outer hair cells. J Neurosci 20:RC116

Brownell WE (2006) The piezoelectric hair cell. In: Eatock RA (ed) Vertebrate hair cells. Springer, New York, pp 313-347

Brownell WE, OGHalai JS (2000) Structural basis of outer hair cell motility or where's the motor? In: Lim D (ed) Cell and molecular biology of the ear. Academic, New York, pp 69-83

Brownell WE, Bader CR, Bertrand D, DE Ribaupierre Y (1985) Evoked mechanical responses of isolated cochlear outer hair cells. Science 227:194-196

Brownell WE, Spector AA, Raphael RM, Popel AS (2001) Micro- and nanomechanics of the cochlear outer hair cell. Annu Rev Biomed Eng 3:169-194

Deak L, Zheng J, Orem A, Du GG, Aguinaga S, Matsuda K, Dallos P (2005) Effects of cyclic nucleotides on the function of prestin. J Physiol 563:483-496

Evans MB, Tonini R, Shope C, Oghalai JS, Jerger JF, Insull WJ, Brownell WE (2006) Dyslipidemia and auditory function. Otol Neurotol 27:609-614

Farrell B, Do Shope C, Brownell WE (2006) Voltage-dependent capacitance of human embryonic kidney cells. Phys Rev E Stat Nonlin Soft Matter Phys 73:041930

Frank G, Hemmert W, Gummer AW (1999) Limiting dynamics of high-frequency electromechanical transduction of outer hair cells. Proc Natl Acad Sci USA 96:4420-4425

Fullekrug J, Simons K (2004) Lipid rafts and apical membrane traffic. Ann N Y Acad Sci 1014:164-169

Helenius A, Aebi M (2001) Intracellular functions of N-linked glycans. Science 291:2364-2369

Huet G, Gouyer V, Delacour D, Richet C, Zanetta JP, Delannoy P, DEgand P (2003) Involvement of glycosylation in the intracellular trafficking of glycoproteins in polarized epithelial cells. Biochimie 85:323-330

Kenworthy AK, Nichols BJ, Remmert CL, Hendrix GM, Kumar M, Zimmerberg J, Lippincott-Schwartz J. (2004) Dynamics of putative Raft-Associated proteins at the Cell surface. J Cell Biol. 165: $735-46$

Liberman MC, Gao J, He DZ, Wu X, Jia S, Zuo J (2002) Prestin is required for electromotility of the outer hair cell and for the cochlear amplifier. Nature 419:300-304

Ludwig J, Oliver D, Frank G, Klocker N, Gummer AW, Fakler B (2001) Reciprocal electromechanical properties of rat prestin: the motor molecule from rat outer hair cells. Proc Natl Acad Sci USA 98:4178-4183

Matsuda K, Zheng J, Du G-G, Klocker N, Madison LD, Dallos P (2004) N-linked glycosylation sites of the motor protein prestin: effects on membrane targeting and electrophysiological function. J Neurochem 89:928-938

Mayor S, Pagano RE (2007) Pathways of clathrin-independent endocytosis. Nat Rev Mol Cell Biol 8:603-612

Navaratnam D, Bai JP, Samaranayake H, Santos-Sacchi J (2005) Nterminal-mediated homomultimerization of prestin, the outer hair cell motor protein. Biophys J 89:3345-3352

NGUYEN TV, BRownell WE (1998) Contribution of membrane cholesterol to outer hair cell lateral wall stiffness. Otolaryngol Head Neck Surg 119:14-20
Oghalai JS, Tran TD, Raphael RM, Nakagawa T, Brownell WE (1999) Transverse and lateral mobility in outer hair cell lateral wall membranes. Hear Res 135:19-28

Oliver D, He DZ, Klocker N, Ludwig J, Schulte U, Waldegger S, Ruppersberg JP, DAllos P, FAKLer B (2001) Intracellular anions as the voltage sensor of prestin, the outer hair cell motor protein. Science 292:2340-2343

ORGAN LE, RAPHAEL RM (2007) Application of fluorescence recovery after photobleaching to study prestin lateral mobility in the human embryonic kidney cell. J Biomed Opt 12:021003

Preyer S, Baisch A, Bless D, Gummer AW (2001) Distortion product otoacoustic emissions in human hypercholesterolemia. Hear Res 152:139-151

Rajagopalan L, Patel N, Madabushi S, Goddard JA, Anjan V, Lin F, Shope C, Farrell B, Lichtarge O, Davidson AL, Brownell WE, Pereira FA (2006) Essential helix interactions in the anion transporter domain of prestin revealed by evolutionary trace analysis. J Neurosci 26:12727-12734

Rajagopalan L, Greeson JN, Xia A, Liu H, Sturm A, Raphael RM, Davidson AL, Oghalai JS, Pereira FA, Brownell WE (2007) Tuning of the outer hair cell motor by membrane cholesterol. J Biol Chem 282:36659-36670

SAITO T, SATO K, SAITo H (1986) An experimental study of auditory dysfunction associated with hyperlipoproteinemia. Arch Otorhinolaryngol 243:242-245

SAnti PA, Mancini P, Barnes C (1994) Identification and localization of the GM1 ganglioside in the cochlea using thin-layer chromatography and cholera toxin. J Histochem Cytochem 42:705-716

Santos-Sacchi J, Kakehata S, Takahashi S (1998) Effects of membrane potential on the voltage dependence of motilityrelated charge in outer hair cells of the guinea-pig. J Physiol 510:225-235

Sfondouris J, Rajagopalan L, Pereira FA, Brownell WE (2008) Membrane composition modulates prestin-associated charge movement. J Biol Chem 283:22473-22841

Sikora MA, Morizono T, Ward WD, Paparella MM, Leslie K (1986) Diet-induced hyperlipidemia and auditory dysfunction. Acta Otolaryngol 102:372-381

Sturm AK, Rajagopalan L, Yoo D, Brownell WE, Pereira FA (2006) Expression and subcellular localization of prestin in cultured cells. Otolaryngol Head Neck Surg 136:434-439

Tami TA, Fankhauser CE, Mehlum DL (1985) Effects of noise exposure and hypercholesterolemia on auditory function in the New Zealand white rabbit. Otolaryngol Head Neck Surg 93:235-239

Trombetta ES, Helenius A (1998) Lectins as chaperones in glycoprotein folding. Curr Opin Struct Biol 8:587-592

Vetrivel KS, Cheng H, Lin W, Sakurai T, Li T, Nukia N, Wong PC, Xu H, Thinakaran G (2004) Association of $\gamma$-secretase with lipid rafts in post-golgi and endosome membranes. J Biol Chem 279:44945-44954

Yanjanin NM, Vélez JI, Gropman A, King K, Bianconi SE, Conley SK, Brewer CC, Solomon B, Pavan WJ, Arcos-Burgos M, Patterson MC, Porter FD (2009) Linear clinical progression, independent of age of onset, in Niemann-Pick disease, type C. Am J Med Genet B Neuropsychiatr Genet (in press)

Yu N, Zhu ML, Zhaо HB (2006) Prestin is expressed on the whole outer hair cell basolateral surface. Brain Res 1095:51-58

Zheng J, Long KB, Shen W, Madison LD, Dallos P (2001) Prestin topology: localization of protein epitopes in relation to the plasma membrane. NeuroReport 12:1929-1935

Zheng J, Shen W, He DZ, Long KB, Madison LD, Dallos P (2000) Prestin is the motor protein of cochlear outer hair cells. Nature 405:149-155

Zheng J, Du GG, Anderson CT, Keller JP, Orem A, Dallos P, Cheatham M (2006) Analysis of the oligomeric structure of the motor protein prestin. J Biol Chem 281:19916-19924 